${ }^{1}$ Department of Ophthalmology, The First Affiliated Hospital, Chongqing Medical University, Chongqing Key Laboratory of Ophthalmology, Chongqing, China

${ }^{2}$ Department of Ophthalmology, Xi'an Central Hospital, Xi'an, China

${ }^{3}$ Department of Ophthalmology, The People's Hospital of Jianyang City, Jianyang, China

${ }^{4}$ Department of Ophthalmology, The Central Hospital of Edors in Inner Mongolia, Edors, China

Correspondence: $X$ Zhang, Department of Ophthalmology, The First Affiliated Hospital, Chongqing Medical University, Chongqing Key Laboratory of Ophthalmology, No. 1 You Yi Road,

Yu Zhong District, Chongqing 400016, China Tel: +86 238901 2704; Fax: + 862389012301 . E-mail: zxued@sina.com ${ }^{5}$ These authors contributed equally to this work.

Received: 4 October 2011 Accepted in revised form: 20 February 2012 Published online: 23 March 2012

\title{
Prevalence and risk factors of diabetic retinopathy in Chongqing pre-diabetes patients
}

Abstract

Purpose To investigate the prevalence of diabetic retinopathy (DR) and relative risk factors among Chongqing pre-diabetes patients. Methods A total of 750 participants were recruited in this cross-sectional study. All participants underwent a complete physical examination and an oral glucose-tolerance test. In all, 110 of the 125 newly diagnosed pre-diabetics and their healthy spouses as controls were examined with fluorescence fundus angiographies, and their blood with biochemical analyses. All the pre-diabetics with DR (23 subjects), 23 normal controls and 23 pre-diabetics without DR were compared for serum concentrations of regulated upon activation, normal T-expressed and secreted (RANTES). Student's $t$-test was used to compare continuous variables, and $\chi^{2}$ test and analysis of variance to compare proportions among groups. Multiple logistic regression models were used to determine the risk factors for DR in pre-diabetics.

Results In all, $20.91 \%$ of the 110 pre-diabetics showed mild non-proliferative DR (NPDR). There was a statistically significant difference in serum concentrations of RANTES between pre-diabetics with and without DR $(P<0.01)$, and also between pre-diabetics with $D R$ and normal controls $(P<\mathbf{0 . 0 1})$. However, age, body mass index, waist-hip ratio, triacylglycerol (TG), total cholesterol (TC), high-density lipoprotein-C, low-density lipoprotein-C, blood urea nitrogen, blood creatinine, and urine albumin excretion rate seemed to have no reliable relationship with DR in pre-diabetics $(P>0.05)$.

Conclusion The prevalence of DR in Chongqing pre-diabetes patients in the study was about $\mathbf{2 0 . 9 1 \%}$ and only mild NPDR was found.
X Chen ${ }^{1,5}$, Y Zhao ${ }^{2,5}$, Z Zhou ${ }^{1}$, X Zhang ${ }^{1}$, Q Li ${ }^{1}$, $\mathrm{L} \mathrm{Bai}^{3}$ and $\mathrm{M}$ Zhang ${ }^{4}$
It seems that RANTES is one possible risk factor associated with DR in pre-diabetics, not age, TG and TC, and others.

Eye (2012) 26, 816-820; doi:10.1038/eye.2012.50; published online 23 March 2012

Keywords: diabetic retinopathy; pre-diabetes; prevalence; RANTES; risk factor

\section{Introduction}

Prevention of type 2 diabetes mellitus and its complications is a major public health challenge because of its large effect on health. Diabetes affected an estimated 171 million people worldwide in 2000, and this number is projected to rise to 366 million by 2030, owing to increases in age, obesity, and urbanization of the world's population. ${ }^{1}$ In addition to this, the prevalence of diabetic retinopathy (DR) is increasing worldwide due to the rising number and prolonged survival of diabetics. ${ }^{2}$ Almost all patients with type 2 diabetes go through one necessary phase called 'pre-diabetes' before the onset of overt diabetes. ${ }^{3}$ Individuals with impaired glucose tolerance and/or impaired fasting glucose are currently considered to have 'pre-diabetes'. ${ }^{4}$ Pre-diabetes not only has the potential risk of developing to diabetes but also can lead to the onset of DR. ${ }^{5}$ DR, a devastating ocular complication of diabetes mellitus, is the leading cause of blindness among working-age adults in the United States and is a serious public health problem throughout the world. ${ }^{5}$ The underlying mechanisms of DR have not been elucidated, although there are several theories. The idea that DR is a low-grade chronic inflammatory condition has gained recent interest. ${ }^{6}$ The chemokine named 
regulated upon activation, normal T-expressed and secreted (RANTES) was proved to be associated with severe DR. ${ }^{7}$ Therefore, in this study, we investigated the prevalence of DR and its risk factors (RANTES specifically included) among pre-diabetes participants, hoping to contribute to the prevention and treatment of DR at an early stage.

\section{Materials and methods}

\section{Study population}

With the assistance of Endocrine Division of the First Affiliated Hospital of Chongqing Medical University, we recruited 421 type 2 diabetic families (2148 subjects) from April 2005 to March 2007 (24 months). From this pool, 750 second-degree relatives of the probands were eligible for this study, after excluding subjects with a history of diabetes, hypertension, cardiac insufficiency, hepatic inadequacy, or kidney dysfunction. All 750 participants underwent a complete physical examination and an oral glucose-tolerance test (OGTT). Of these, 110 newly diagnosed pre-diabetics and their healthy spouses (who were treated as controls) had a detailed ophthalmic examination (FFA included) and blood biochemical analyses. Then, a total of 23 members (20.91\% of the 110 pre-diabetics) with DR, 23 pre-diabetics without DR and 23 healthy age-matched controls were examined for serum RANTES levels. Our study followed the Declaration of Helsinki Principles and was approved by the Ethics Committee of Chongqing Medical University (the Institutional Review Board). Informed consent was obtained from each participant.

\section{Oral glucose-tolerance test}

The 750 participants were instructed to maintain their usual physical activity and diet for at least 3 days before OGTT. After at least $10 \mathrm{~h}$ of overnight fasting, they were challenged with the oral glucose load used in the standardized OGTT. Pre-diabetes was diagnosed based on the American Diabetes Association criteria; ${ }^{8}$ thus 125 pre-diabetes subjects were newly diagnosed.

\section{Fluorescence fundus angiographies}

For ophthalmic examination, $1 \%$ tropicamide was administered to both eyes until the best possible mydriasis was obtained. Fortunately, all 125 newly diagnosed pre-diabetics were free of disorders such as glaucoma, uveitis, keratitis, retinal detachment, and heavy cataract that may distort the FFA conclusions. Among those, 110 pre-diabetics and their healthy spouse controls were examined with FFA, using a TOPCON
TRC, 50IX retinal camera (Topcon, Tokyo, Japan). The other 15 pre-diabetics did not receive FFA examination because of allergy to fluorescein sodium or worries about its side effects. The assessment of retinopathy was done by only one retinal specialist, using FFA images. DR was classified using a severity scale recommended by the International Council of Ophthalmology and American Academy of Ophthalmology (Table 1). ${ }^{9}$

\section{Systemic and blood assessment}

A standard questionnaire ${ }^{10}$ administered by trained staff was used to obtain information on name, sex, age, and history of diseases. The weights of the subjects were measured and recorded to the nearest kilograms and the heights to the nearest centimeters. Body mass index (BMI) and waist-hip ratio (WHR) were calculated by their respective standard equations. Blood samples were drawn after a 10 -h overnight fasting by the 110 pre-diabetes subjects and their healthy spouses. Then biochemical analyses were conducted with an OLYMPUS AU5400 Full Automatic Biochemical Analyzer (Olympus Corp., Tokyo, Japan). End points included triacylglycerol (TG), total cholesterol (TC), highdensity lipoprotein-C (HDL-C), low-density lipoproteinC (LDL-C), blood urea nitrogen (BUN), and blood creatinine. The 2 -h random urine albumin excretion rate (UAER) was measured with radioimmunoassay. The serum concentration of RANTES was measured using Enzyme-Linked Immunosorbent Assay (RANTES Instant ELISA, Bender Medsystems, Vienna, Austria).

Table 1 Diabetic Retinopathy Severity Scale

\begin{tabular}{ll}
\hline Severity level & Findings observable upon dilated ophthalmoscopy \\
\hline $\begin{array}{l}\text { No apparent } \\
\text { retinopathy }\end{array}$ & No abnormalities \\
$\begin{array}{l}\text { Mild NPDR } \\
\text { Moderate NPDR }\end{array}$ & $\begin{array}{l}\text { Microaneurysms only } \\
\text { but less than severe form }\end{array}$ \\
Severe NPDR & $\begin{array}{l}\text { Any of the following and no signs of } \\
\text { proliferative retinopathy } \\
\text { More than 20 intraretinal hemorrhages } \\
\text { in each of the four quadrants } \\
\text { Definite venous beading in two or } \\
\text { more quadrants } \\
\text { Prominent intraretinal microvascular } \\
\text { abnormality (IRMA) in one or more } \\
\text { quadrants }\end{array}$ \\
& $\begin{array}{l}\text { One or both of the following } \\
\text { Neovascularization }\end{array}$ \\
& Vitreous hemorrhage/preretinal \\
hemorrhage
\end{tabular}

Abbreviations: NPDR, non-proliferative diabetic retinopathy; PDR, proliferative diabetic retinopathy. 


\section{Data analysis}

Statistical analysis was carried out using SPSS 13 (SPSS Inc., Chicago, IL, USA). Student's $t$-test was used to compare continuous variables such as age, gender, and BMI (Table 3). $\chi^{2}$ test and one-way analysis of variance were used to compare proportions among groups as in

Table 2 Prevalence of DR in pre-diabetics and their healthy spouse controls

\begin{tabular}{lccc}
\hline & $D R$ & NDR & Ratio (\%) \\
\hline Normal controls & $0(11.5)$ & $110(98.5)$ & 0 \\
Pre-diabetics & $23(11.5)$ & $87(98.5)$ & 20.91 \\
\hline
\end{tabular}

Abbreviations: DR, diabetic retinopathy (NPDR specifically referred, as more severe DR was not found); NDR, non-diabetic retinopathy.

The numerals in the brackets are the theoretical frequency and the numerals out of the brackets are the actual frequency. $\chi^{2}=28.6852, P<0.05$

Table 3 Clinical features of subjects with pre-diabetes and spouse controls

\begin{tabular}{lccc}
\hline Variables & $\begin{array}{c}\text { With } \\
\text { pre-diabetes }\end{array}$ & $\begin{array}{c}\text { Normal } \\
\text { controls }\end{array}$ & P-value \\
\hline Age (year) & $50.33 \pm 12.62$ & $53.07 \pm 17.62$ & 0.596 \\
Gender $(\mathrm{male} /$ female $)$ & $45 / 65$ & $43 / 67$ & 0.386 \\
BMI $\left(\mathrm{kg} / \mathrm{m}^{2}\right)$ & $23.42 \pm 2.95$ & $23.77 \pm 2.82$ & 0.703 \\
WHR & $0.87 \pm 0.05$ & $0.85 \pm 0.06$ & 0.296 \\
TG $(\mathrm{mmol} / \mathrm{l})$ & $1.38 \pm 0.60$ & $1.29 \pm 0.50$ & 0.57 \\
TC $(\mathrm{mmol} / \mathrm{l})$ & $5.26 \pm 0.86$ & $5.34 \pm 0.93$ & 0.773 \\
HDL-C $(\mathrm{mmol} / \mathrm{l})$ & $1.50 \pm 0.31$ & $1.65 \pm 0.38$ & 0.179 \\
LDL-C $(\mathrm{mmol} / \mathrm{l})$ & $3.02 \pm 0.64$ & $2.96 \pm 0.70$ & 0.781 \\
BUN $(\mathrm{mmol} / \mathrm{l})$ & $5.51 \pm 1.42$ & $5.72 \pm 1.61$ & 0.681 \\
Blood creatinine $(\mu \mathrm{mol} / \mathrm{l})$ & $74.71 \pm 16.76$ & $70.07 \pm 13.97$ & 0.348 \\
UAER $(\mu \mathrm{g} / \mathrm{min})$ & $309.96 \pm 86.22$ & $303.18 \pm 98.90$ & 0.829 \\
\hline
\end{tabular}

Abbreviations: BUN, blood urea nitrogen; HDL-C, high-density lipoprotein-C; LDL-C, low-density lipoprotein-C; TC, total cholesterol; TG, triacylglycerol; UAER, urine albumin excretion rate; WHR, waist-hip ratio.

BMI $=$ weight $(\mathrm{kg}) /$ height $\left(\mathrm{m}^{2}\right)$
Table 2 and Table 5, respectively. $P<0.05$ was accepted as statistically significant. Multiple logistic regression analysis was performed (Table 4), using DR as the dependent variable. Age, BMI, WHR, TG, TC, HDL-C, LDL-C, BUN, blood creatinine, and UAER were entered into a logistic regression model to determine independent predictors of DR. Odds ratios and 95\% confidence intervals are presented.

\section{Results}

A total of 125 pre-diabetics among the 750 participants were newly diagnosed. In all, 20.91\% (23/110) of prediabetics showed mild non-proliferative DR (NPDR), while none of the controls had apparent DR (Table 2). The difference was statistically significant $(P<0.05)$. Table 3 shows no statistical difference between prediabetics and normal controls, with regard to age, gender, BMI, WHR, TG, TC, HDL-C, LDL-C, BUN, blood creatinine, and UAER. Table 4 shows that age, BMI, WHR, TG, TC, HDL-C, LDL-C, BUN, blood creatinine, and UAER had no meaningful relationship with DR in pre-diabetics $(P>0.05)$. As evidenced in Table 5, there did exist a significant difference in serum RANTES concentrations between pre-diabetics with and without DR $(P<0.01)$, and also between pre-diabetics with DR and normal controls $(P<0.01)$. Nevertheless, there was no significant difference between normal controls and pre-diabetics without DR $(P>0.05)$.

\section{Discussion}

The prevalence of DR in Chongqing pre-diabetes patients in this study was $20.91 \%$ (23/110), which was higher than that in their healthy spouse controls. Also, DR prevalence rate in our study was higher than those reported by Gabir et $a l^{11}$ and Nagi et $a l^{12}$ which were 1.2 and $12 \%(8 / 68)$, respectively. The lower prevalence of DR in

Table 4 Multiple logistic regression analysis of factors for DR in pre-diabetics

\begin{tabular}{lccccc}
\hline Variables & $\beta$ & $S E$ & P-value & OR & 95\% CI \\
\hline Age (year) & -0.028 & 0.029 & 0.349 & 0.973 & $0.919-1.031$ \\
BMI $\left(\mathrm{kg} / \mathrm{m}^{2}\right)$ & -0.520 & 0.269 & 0.053 & 0.595 & $0.351-1.007$ \\
WHR & 30.151 & 15.542 & 0.052 & $1 \mathrm{E}+013$ & $0.732-2 \mathrm{E}+026$ \\
TG $(\mathrm{mmol} / \mathrm{l})$ & -1.251 & 1.466 & 0.394 & 0.286 & $0.016-5.067$ \\
TC $(\mathrm{mmol} / \mathrm{l})$ & 0.766 & 2.018 & 0.704 & 2.152 & $0.041-112.271$ \\
HDL-C (mmol/l) & -2.318 & 2.807 & 0.409 & 0.098 & $0.000-24.141$ \\
LDL-C $(\mathrm{mmol} / \mathrm{l})$ & 0.233 & 2.100 & 0.911 & 1.263 & $0.021-77.360$ \\
BUN $(\mathrm{mmol} / \mathrm{l})$ & -0.077 & 0.322 & 0.811 & 0.926 & $0.492-1.740$ \\
Blood creatinine $(\mu \mathrm{mol} / \mathrm{l})$ & 0.008 & 0.034 & 0.810 & 1.008 & $0.943-1.078$ \\
UAER $(\mu \mathrm{g} / \mathrm{min})$ & -0.005 & 0.006 & 0.422 & 0.995 & $0.983-1.007$ \\
\hline
\end{tabular}

Abbreviations: CI, confidence interval; OR, odds ratio; SE, stand error

$\beta$ stands for maximum likelihood estimates of regression coefficient. 
Table 5 Serum concentrations of RANTES between Group 1: normal controls, Group 2: pre-diabetics without DR, and Group 3: pre-diabetics with DR

\begin{tabular}{lcccc}
\hline Group & Concentrations $(p g / l)$ & \multicolumn{3}{c}{ P-value } \\
\cline { 3 - 5 } & & 1 & 2 & 3 \\
\hline 1 & $21402.67 \pm 3231.18$ & - & $>0.05$ & $<0.01$ \\
2 & $20939.57 \pm 3749.73$ & $>0.05$ & - & $<0.01$ \\
3 & $35368.30 \pm 5590.30$ & $<0.01$ & $<0.01$ & - \\
\hline
\end{tabular}

Abbreviation: RANTES, regulated upon activation, normal T-expressed and secreted.

the two studies might be attributed to the methods of retinal assessment. In the study by Gabir et al, subjects were examined by observing their dilated pupils using a direct ophthalmoscope, and in the study by Nagi et al, by fundus photography after mydriasis. But in our study, subjects were examined by FFA, which is apparently advantageous in detecting DR. In addition, small sample sizes and different grading criteria of DR in these three studies might be taken into consideration.

Earlier studies have shown that age, ${ }^{13,14}$ gender, ${ }^{14,15}$ BMI, ${ }^{16,17}$ WHR, ${ }^{16}$ TC, $^{13}$ HDL-C, ${ }^{18}$ LDL-C, ${ }^{19}$ BUN, blood creatinine, ${ }^{20}$ and $\mathrm{UAER}^{21,22}$ have certain associations with DR in diabetics. However, in our study, it appears that those related above are not risk factors for DR in prediabetics. These discrepancies might have resulted from the fact that metabolism disorders are much lighter in pre-diabetics than in diabetics, as Table 3 suggests no significant difference between pre-diabetics and healthy spouse controls in clinical characteristics such as BMI, TC, and LDL-C. However, the discrepancies may give us a new reflection on the risk factors for DR. Besides the traditional factors like BMI, TC, and TG that are associated with DR in diabetics, there are probably other factors too, as DR also exists in pre-diabetics without such known factors. Perhaps the hyperglycemia-induced inflammatory response can be counted.

In the pathogenesis of DR, an accumulating body of evidence shows that immunological mechanisms characterized by many features typical of inflammation have a prominent role. The upregulation of cytokines and other inflammatory mediators led to an influx of leukocytes and persistent low-grade inflammation. The cytokines and mediators are said to contribute actively to DR-associated damage to the retinal vasculature and neovascularization. ${ }^{23}$ RANTES produced by inflammatory cells, retinal endothelial cells, and pigment epithelial cells ${ }^{24}$ is a member of the chemokine family. Meleth et $\mathrm{al}^{7}$ showed that RANTES levels largely increased in subjects with severe NPDR versus less severe NPDR $(P<0.001)$, but RANTES levels unexpectedly decreased in subjects with less severe
NPDR versus normal controls. Thus, Meleth believes that the latter may not correctly reflect the real variation. However, in our study, serum levels of RANTES were significantly elevated in pre-diabetics with DR compared with pre-diabetics without DR and normal controls $(P<0.01)$, while RANTES levels were not significantly different between pre-diabetics without DR and normal controls $(P>0.05)$. Taken together, RANTES may be associated with mild NPDR in pre-diabetics and has a potential role in the development of DR; the concrete mechanism needs further research.

Our study has certain limitations. First, this was a survey based on family investigation rather than a population-based study. However, the prevalence of pre-diabetes in our study (125/750) was similar to that reported in China (15.5\%), ${ }^{25}$ which justifies our further survey on DR prevalence. But the eligible sample size was small for pre-diabetics (125) and even smaller for pre-diabetics with DR (23), which may lessen our conclusions' reliability, stability, and representativeness. Second, the population inclusion criterion was tough, as we excluded persons with diabetes, hypertension, cardiac failure, and other related diseases. We aimed to avoid retinal microaneurysms (standard of DR diagnosis) that are caused by diabetes, hypertension, or other possible illnesses ${ }^{17,18}$ so that we could get a relatively accurate prevalence of DR in pre-diabetics. However, doing this might also make our population seem healthier than those of other studies, and accordingly weaken the comparability between our study and other similar studies. ${ }^{12,26}$ Third, we could not research all possible risk factors including gender, ${ }^{14,15}$ duration of pre-diabetes, and hyperglycemia-related inflammatory factors SDF- $1 \alpha$, ICAM-1, and MCP- $1 .{ }^{7}$ Therefore, perhaps we have missed some potential risk factors. The strength of our study lies in that we performed OGTT and FFA to get creditable diagnoses of pre-diabetes and DR.

The data obtained may be valuable for further study and comparisons between relative works: for instance, the Diabetes Prevention Program ${ }^{27}$ and the study by Dalton, ${ }^{26}$ both of which indicate that $8 \%$ of pre-diabetes patients have DR. Meanwhile, the controls were selected from among the healthy spouses of the newly diagnosed pre-diabetics, and hence the confounding factors have been reduced as much as possible.

In summary, the prevalence of DR in Chongqing pre-diabetes patients in our study was $20.91 \%$ and only mild NPDR was detected; more severe DR was not found. RANTES, an inflammatory factor related to hyperglycemia, may be one possible risk factor for the incidence and progression of $\mathrm{DR}$ in pre-diabetics. However, age, BMI, WHR, TG, TC, HDL-C, LDL-C, BUN, blood creatinine, and UAER show no significant association with DR in pre-diabetics. 


\section{Summary}

\section{What was known before}

- A total of $8 \%$ of pre-diabetes patients had DR reported by the Diabetes Prevention Program using fundus photography, and some risk factors related were identified.

\section{What this study adds}

- Prevalence of DR in Chongqing pre-diabetes persons was $20.91 \%$ by FFA examination, and regulated upon activation, normal T-expressed and secreted (RANTES) may be listed as one risk factor of DR in pre-diabetics.

\section{Conflict of interest}

The authors declare no conflict of interest.

\section{Acknowledgements}

This work was supported by a Grant-in-Aid for scientific research from the Endocrine Division of the First Affiliated Hospital of Chongqing Medical University. We are indebted to all participants of the study.

\section{References}

1 Diabetes Prevention Program Research Group. 10-Year follow-up of diabetes incidence and weight loss in the Diabetes Prevention Program Outcomes Study. Lancet 2009; 374: 1677-1686.

2 Aylward GW. Progressive changes in diabetics and their management. Eye 2005; 19: 1115-1118.

3 Kawamori R. Primary prevention of diabetes-importance of early diagnosis and interventions in IGT \& IFG. Nippon Rinsho 2005; 63(Suppl 2): 49-54.

4 American Diabetes Association. Tests of glycemia in diabetes (Position Statement). Diabetes Care 2001; 24 (Suppl 1): S80-S82.

5 Wright AD, Dodson PM. Diabetic retinopathy without diabetes. Diabet Med 2007; 24(10): 1174; (author reply 1174-1175).

6 Ali TK, El-Remessy AB. Diabetic retinopathy: current management and experimental therapeutic targets. Pharmacotherapy 2009; 29(2): 182-192.

7 Meleth AD, Agrón E, Chan CC, Reed GF, Arora K, Byrnes G et al Serum inflammatory markers in diabetic retinopathy. Invest Ophthalmol Vis Sci 2005; 46(11): 4295-4301.

8 American Diabetes Association. Report of the expert committee on the diagnosis and classification of diabetes mellitus. Diabetes Care 2003; 26(Suppl1): S5-S20.

9 Wilkinson CP, Ferris 3rd FL, Klein RE, Lee PP, Agardh CD, Davis $\mathrm{M}$ et al. Proposed International Clinical Diabetic Retinopathy and Diabetic Macular Edema Disease Severity Scales. Ophthalmology 2003; 110: 1677-1682.

10 Luepker RV, Evans A, McKeigue P, Reddy KS Cardiovascular Survey Methods, 3rd edn. World Health Organization: Geneva, 2004.

11 Gabir MM, Hanson RL, Dabelea D, Imperatore G, Roumain J, Bennett PH et al. Plasma glucose and prediction of microvascular disease and mortality: evaluation of 1997 American Diabetes Association and 1999 World Health Organization criteria for diagnosis of diabetes. Diabetes Care 2000; 23(8): 1113-1118.

12 Nagi DK, Pettitt DJ, Bennett PH, Klein R, Knowler WC. Diabetic retinopathy assessed by fundus photography in Pima Indians with impaired glucose tolerance and NIDDM. Diabet Med 1997; 14(6): 449-456.

13 Wong TY, Cheung N, Tay WT, Wang JJ, Aung T, Saw SM et al. Prevalence and risk factors for diabetic retinopathy: the Singapore Malay Eye Study. Ophthalmology 2008; 115(11): 1869-1875.

14 Peng XY, Wang FH, Liang YB, Wang JJ, Sun LP, Peng Y et al. Retinopathy in persons without diabetes: the Handan Eye Study. Ophthalmology 2010; 117(3): 531-537; 537.e1-e2.

15 Varma R, Macias GL, Torres M, Klein R, Peña FY, Azen SP, Los Angeles Latino Eye Study Group. Biologic risk factors associated with diabetic retinopathy: the Los Angeles Latino Eye Study. Ophthalmology 2007; 114(7): 1332-1340.

16 Chao JR, Lai MY, Azen SP, Klein R, Varma R, Los Angeles Latino Eye Study Group. Retinopathy in persons without diabetes: the Los Angeles Latino Eye Study. Invest Ophthalmol Vis Sci 2007; 48(9): 4019-4025.

17 Jeganathan VS, Cheung N, Tay WT, Wang JJ, Mitchell P, Wong TY. Prevalence and risk factors of retinopathy in an Asian population without diabetes: the Singapore Malay Eye Study. Arch Ophthalmol 2010; 128(1): 40-45.

18 Xie XW, Xu L, Wang YX, Jonas JB. Prevalence and associated factors of diabetic retinopathy: The Beijing Eye Study 2006. Graefes Arch Clin Exp Ophthalmol 2008; 246(11): 1519-1526.

19 Sachdev N, Sahni A. Association of systemic risk factors with the severity of retinal hard exudates in a north Indian population with type 2 diabetes. J Postgrad Med 2010; 56(1): 3-6.

$20 \mathrm{Hu} H Y$, Lu B, Zhang ZY, Mao LY, Song XY, Dong XH et al. An epidemiological study on diabetic retinopathy among type 2 diabetic patients in Shanghai. Zhonghua Liu Xing Bing Xue Za Zhi 2007; 28(9): 838-840.

21 Dowse GK, Humphrey AR, Collins VR, Plehwe W, Gareeboo H, Fareed D et al. Prevalence and risk factors for diabetic retinopathy in the multiethnic population of Mauritius. Am J Epidemiol 1998; 147(5): 448-457.

22 Migdalis IN, Hatzigakis SM, Dimakopoulos N, Tolis A, Likoudi A. Diabetic retinopathy and urinary albumin excretion rate in type II (non-insulin-dependent) diabetic patients. J Med 1996; 27(5-6): 341-350.

23 Adamis AP, Berman AJ. Immunological mechanisms in the pathogenesis of diabetic retinopathy. Semin Immunopathol 2008; 30(2): 65-84.

24 Crane IJ, Wallace CA, Mckilllop smith S, Forrester JV. Control of chemokine production at the blood-retinal barrier. Immunology 2000; 101: 426-433.

25 Yang W, Lu J, Weng J, Jia W, Ji L, Xiao J et al. Prevalence of diabetes among men and women in China. $N$ Engl J Med 2010; 362: 1090-1101.

26 Dalton M. Diabetic retinopathy found in $8 \%$ of pre-diabetic patients. Ocular Surg News 2005; 23: 41.

27 Diabetes Prevention Program Research Group. The prevalence of retinopathy in impaired glucose tolerance and recent-onset diabetes in the Diabetes Prevention Program. Diabet Med 2007; 24: 137-144. 